\title{
On the Impact of Probabilistic Shaping on Fiber Nonlinearities
}

This paper was downloaded from TechRxiv (https://www.techrxiv.org).

\section{LICENSE}

CC BY-NC-SA 4.0

SUBMISSION DATE / POSTED DATE

$17-12-2021$ / 03-02-2022

\section{CITATION}

Tanha, Ahmad; Rabbani, Hami; Beygi, Lotfollah (2021): On the Impact of Probabilistic Shaping on Fiber Nonlinearities. TechRxiv. Preprint. https://doi.org/10.36227/techrxiv.17260178.v2

$\mathrm{DOI}$ 


\title{
On the Impact of Probabilistic Shaping on Fiber Nonlinearities
}

\author{
A. Tanha, H. Rabbani and L. Beygi* \\ K. N. Toosi University of Technology, Tehran, Iran
}

\section{ARTICLE INFO}

\section{Keywords:}

Probabilistic shaping

Gaussian noise models

Optical communications

Constant composition distribution matcher

\begin{abstract}
A B S TR ACT
This paper addresses probabilistic shaping (PS) which has been a latest key technique to approach capacity of fiber-optic channels. We investigate the impact of PS on nonlinear interference (NLI), including self channel interference (SCI), cross channel interference (XCI), and multi channel interference (MCI) for a polarization multiplexed 16-ary quadrature amplitude modulation format in a wavelength division multiplexed (WDM) system. To this end, we consider performing PS in two scenarios: (i) Solely on the channel of interest and (ii) over all C-band WDM channels of a fiber-optic link by analyzing the effective signal to noise ratio and symbol error rate.

It is demonstrated that using the enhanced Gaussian noise model with merely $10 \%$ overhead in the first scenario, the applied PS scheme increases the SCI and the total experienced NLI by about $19.23 \%$, and $6.6 \%$, respectively. Interestingly, despite enhancing the NLI in this scenario, the simulated PS technique leads to about $47.6 \%$ increase in the transmission reach. In the second scenario, the numerical results show increase of the SCI, XCI, and total NLI around $19.8 \%, 23.34 \%$, and $20.2 \%$, respectively, but resulting in an increase of $32.3 \%$ in the transmission reach.
\end{abstract}

\section{Introduction}

Today's society relies on fast and reliable exchange of information [1]. The extreme growth in the demand for data usage of numerous emerging services and applications such as cloud computing, social medias, etc. are stretching the needs for high-capacity communications [1]. The increase in traffic demand together with the development of softwaredefined transceivers that can adapt the transmission parameters to the physical channel have increased the interest in designing networks that utilize the resources more efficiently [2]. High prices of the equipments which are used in the backbone of optical networks motivates usage of the digital signal processing (DSP) techniques with reasonable complexity on the same hardwares. One of these techniques which significantly improves the performance of the network is probabilistic shaping (PS). PS is used in optical communications to increase signal to noise ratio (SNR) per symbol for a fixed launch power that provides a substantial increase in the transmission reach. It also furnishes fine adaptation in the transmission rate.

\subsection{Problem Statement}

To study the influence of PS on the nonlinear effects in fiber-optic communications, a comprehensive knowledge about the channel impairments is inevitable. Channel models which accurately compute physical layer impairments are useful tools to analytical elaborate on non-linear interference (NLI) noise terms, consisting of self channel interference (SCI), cross channel interference (XCI), and multi channel interference (MCI), instead of computationally complex numerical methods such as split-step Fourier method (SSFM).

Investigating the effect of PS on each nonlinear term of

${ }^{*}$ Corresponding author

Email Addresses:

@ahmadtanha@email.kntu.ac.ir (A. Tanha);

hami. rabbani@email.kntu.ac.ir (H. Rabbani); beygi@kntu.ac.ir (L. Beygi) $\operatorname{ORCID}(\mathrm{s})$
NLI is beneficial for designing NLI-optimized modulationformats. Furthermore, analyzing the effect of PS on WDM transmission systems with respect to transmitter and link parameters is crucial. However, these two topics have not been addressed before in literature.

\subsection{Related Work}

Main recent performance improvement technique for fiber-optical communications extensively discussed in the literature is probabilistic signal shaping. Shaping is performed either with changing position of constellation points to make a Gaussian distribution in complex plane, called geometric shaping (GS), or with changing probability of constellation symbols, called PS. GS has some disadvantages in practice where mentioned thoroughly in [3]: (i) there is no simple solution to finding locations of the constellation points for arbitrary channel conditions; (ii) the irregular constellation points of GS increase the complexity of coherent DSP for robust signal recovery prior to decoding; (iii) the general infeasibility of Gray mapping increases the complexity of demapping symbols to soft-decision bit metrics. Unlike GS, PS typically is used in a fixed architecture where introduced in [4] including PS and channel coding jointly aka probabilistic amplitude shaping architecture. It enables to use different forward error correction schemes with the same PS encoder. It also allows to use the same structure for the receiver and does not need to involve in the receiver design like GS.

A comprehensive review on the evolution of fiber-optic channel modeling can be found in [5], so we only review here some of the well-known models introduced in the literature. Gaussian noise (GN) model was proposed in [6] is capable of estimating uncompensated optical coherent transmission system performance. However its predictions, when used to obtain a detailed picture of NLI noise accumulation along a link, may be affected by a substantial NLI overestimation error, especially in the first spans of the link [6]. Enhanced Gaussian noise (EGN) model of [7] includes well-known impairments made by fiber-optic channel which degrade per- 


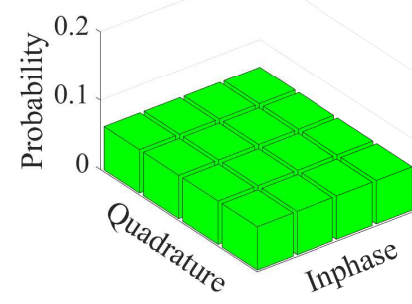

(a) $H\left(P_{1}\right)=4$

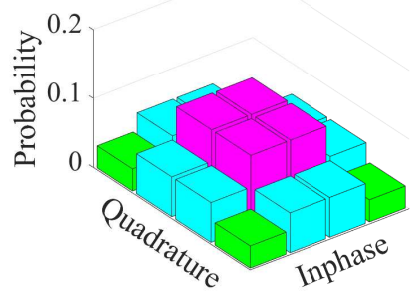

(b) $H\left(P_{2}\right)=3.8807$

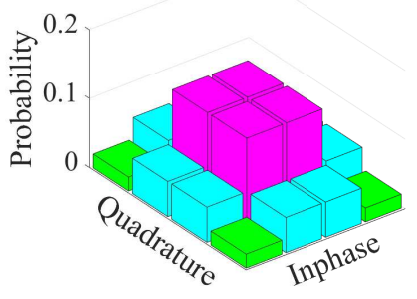

(c) $H\left(P_{3}\right)=3.7208$

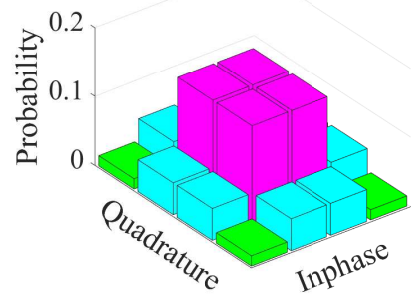

(d) $H\left(P_{4}\right)=3.6008$

Figure 1: An intuitive instance of PS. The bars represent the probability of each constellation symbol. These distributions made by CCDM on a 16-QAM constellation.

formance of transmission system. Actually the correction terms appeared in it was added to the GN model terms where provide a better range of precision.

The concept of PS was introduced by three independent groups $[8,9,10]$ in the form of shell mapping technique in the same time in 1990's. Shell mapping is specified in the ITU-T Recommendation V.34 standard for constellation shaping [11] and it is widely used in wireless modems. Although PS has advantageous applications in wireless communications, it also plays an important role in fiber-optic communications. Lots of research papers studied PS in optical transmission systems in recent years, e.g $[12,13,14,3$, $15,16,17]$. More detailed, a pragmatic rate-adaptive probabilistically shaped coded modulation scheme intorduced in [12]. A PS scheme proposed for optical wavelength division multiplexed (WDM) systems in [13] and a reach improvement of around 7\% reported. It has been experimentally shown in [15] that PS increases transmission reach by more than $40 \%$. It also has been demonstrated using numerical simulations together with GN model and EGN model in [16], [17], respectively that PS reduces effective SNR after fiber propagation in a fixed average launch power, while it increases achievable information rates (AIRs). Rate/reach tradeoff via PS has been investigated in flexible optical transceivers in [14].

All of the aforementioned works investigated PS using information-theoretic approach by measuring MI and AIRs in a coded system. We consider an uncoded system and use digital communications fundamentals for analytical study of the following problems.

\subsection{Main Contributions}

Considering the nonlinear behavior of optical channels, we investigate the effect of PS on different NLI noise terms of fiber nonlinearities by using the EGN model in a polarization multiplexed (PM) 16-ary quadrature amplitude modulation (QAM) WDM system. We also measure two figures of merit for comparing different schemes: effective SNR and symbol error rate (SER). Moreover, we answer two main questions: (i) How much does PS effect on each term of NLI and which of them is more influenced by PS? (ii) What difference would be obtained by applying PS to all WDM channels compare to the case that PS is applied merely to the channel of interest (COI)?

The rest of this paper is organized as follows. In Section 2, PS is discussed in detail. In Section 3, EGN model of fiber-optic channel is explained. In Section 4, performance assessment metrics are introduced. After that, numerical results are provided in Section 5 and finally concluding remarks are stated in Section 6.

\section{Probabilistic Shaping}

PS is used as a technique in fiber-optic systems in order to change the probabilities of modulation symbols that leads to reducing average transmitted power where causes a dramatic improvement of system performance. PS also decreases the gap to the Shannon limit and increases optical channel capacity and transmission reach. The desired distribution in PS is chosen from Maxwell-Boltzmann (MB) family that is a Gaussian like and well known distribution in physics and statistical mechanics. MB probability distribution is given by $[8$, Section IV]

$$
P_{X}(x)=\frac{e^{-\lambda|x|^{2}}}{\sum_{i=1}^{M} e^{-\lambda\left|x_{i}\right|^{2}}}
$$

where $x$ represents constellation symbols which are the channel inputs, $M$ is the modulation order, and $\lambda$ is the shaping factor that plays an important role in the trade-off between bit rate and average energy. The scalar $\lambda$ is chosen such that the mutual information (MI) between a MB-distributed input and the corresponding continuous channel output is maximized for an additive white Gaussian noise (AWGN) channel at a particular SNR [17].

Four different probability distribution of probabilistically shaped 16-QAM are shown in Figure 1. It is obvious that the probability of symbols with lower energy is larger than the probability of symbols with higher energy due to PS. As distributions become more shaped, the entropies decrease.

One of the most used methods of PS is called constant compositon distribution matching (CCDM) where proposed in [18] and belongs to fixed-to-fixed (f2f) length distribution matcher (DM) family. It transforms sequences of $k$ uniformly distributed bits to sequences of $n$ nonuniformly distributed symbols. 
The main idea behind CCDM is minimizing informational divergence that is also known as Kullback-Leibler divergence or relative entropy between the desired distribution and the distribution made by CCDM. The name of CCDM recalls that all of the outputs of this kind of DM is constant composition and the sequence is called $n$-type, i.e. the number of occurences of each output alphabet symbol has a specific value that can be computed using [19, Algorithm 2].

The significant advantage of CCDM in compare with earlier DMs is the fact that it is asymptotically optimal, i.e. when the length of the output of DM is very large, the rate of DM converges to the entropy of the distribution. In the other words, we have $\frac{k}{n}=H(P)$ when $n \rightarrow \infty$.

Sequences $B^{k}=B_{1} B_{2} \ldots B_{k}$ and $A^{n}=A_{1} A_{2} \ldots A_{n}$ are input and output blocks of the DM, respectively. The empirical distribution of a sequence $\mathbf{u}=u_{1} u_{2} \ldots u_{n}$ of length $n$ is defined as [18, Section III]

$$
P_{\mathbf{u}}(\ell)=\frac{n_{\ell}(\mathbf{u})}{n},
$$

where $n_{\ell}(\mathbf{u})=\left|\left\{i: u_{i}=\ell\right\}\right|$ is the number of occurences of symbol $\ell$ in sequence $\mathbf{u}$. The set of sequences with empirical distribution $P$ of length $n$ is then given by [18, Section III-A]

$$
\mathcal{T}_{P}^{n}=\left\{\boldsymbol{\vartheta} \in \mathcal{A}^{n} \mid \frac{n_{\ell}(\boldsymbol{\vartheta})}{n}=P(\ell) \quad \forall \ell \in \mathcal{A}\right\},
$$

where $\mathcal{A}$ is the alphabet of the output of DM which corresponds to the set of possible signal amplitudes in amplitude shift keying (ASK). The mapping to a set of vectors defined by $\mathcal{T}_{P}^{n}$ is done using the function $f_{\mathrm{CCDM}}$ where uses arithmetic coding for indexing sequences efficiently and makes codewords.

$$
f_{\mathrm{CCDM}}:\{0,1\}^{k} \rightarrow \mathcal{T}_{P}^{n} .
$$

To ensure the possibility of inverting transformation functionality of DM, input blocklength of CCDM can be at most $\log _{2}\left|\mathcal{T}_{P}^{n}\right|$. It set to $k=\left\lfloor\log _{2}\left|\mathcal{T}_{P}^{n}\right|\right\rfloor$. The function $f_{\mathrm{CCDM}}$ is invertible and a codebook that is constructed in this manner is stored and the demapper uses it in the receiver. A ready to use MATLAB web demo package of CCDM where proposed in [20] is used in this work.

\section{Estimation of the fiber nonlinearities}

To analyze fiber-optic communications, a high accuracy AWGN model including all impairments that recently remarkably has been studied is a promising solution instead of computationally complex numerical methods such as SSFM. EGN model is the most accurate model has been presented. It is used for simulating an optical link with the desired characterestics and its predictions are such accurate where is used in the optical networks design and analysis problems.

The impairments with this model are divided to three segments. $1^{s t}$ segment is the impact of the channel of interest
(COI) on itself which is called SCI. When two or more optical channels are transmitted simultaneously inside an optical fiber using the WDM technique, another nonlinear terms are appeard. $2^{\text {nd }}$ segment is the result of interference between the COI and each of the interfering (INT) channels, namely XCI. $3^{\text {rd }}$ segment is caused by simultaneously interaction of the COI with two or more INT channels which is known as MCI. SCI and XCI make significant degradation in system performance and $\mathrm{MCI}$ is often ignored because of its small amount, despite all of them is studied in this paper. EGN model of [5] without considering stimulated raman scattering is used in this work. It is approximated using Monte Carlo method of [21].

NLI can be interpreted as a noise with the following variance

$$
\sigma_{\mathrm{NLI}}^{2}=\int_{-\frac{R_{s}}{2}}^{\frac{R_{s}}{2}}\left[G_{\mathrm{SCI}}^{\mathrm{EGN}}(f)+G_{\mathrm{XCI}}^{\mathrm{EGN}}(f)+G_{\mathrm{MCI}}^{\mathrm{EGN}}(f)\right] \mathrm{d} f,
$$

where $G_{\mathrm{SCI}}^{\mathrm{EGN}}(f), G_{\mathrm{XCI}}^{\mathrm{EGN}}(f)$, and $G_{\mathrm{MCI}}^{\mathrm{EGN}}$ are power spectral density of each impairment term (SCI, XCI, \& MCI), respectively which can be extracted from [5] or [7]. Since NLI variance is power dependent and it would be better for comparison, if a same situation be in all WDM channels, it is assumed that the input power of both of the COI and INT channels are equal, i.e. $P_{\mathrm{L}, \mathrm{COI}}=P_{\mathrm{L}, \mathrm{INT}}=P_{\mathrm{L}}$, where $P_{\mathrm{L}}=$ $\mathbb{E}\left\{\left|a_{\mathrm{x}}\right|^{2}+\left|a_{\mathrm{y}}\right|^{2}\right\}$ is the average power of the constellations of dual polarization. Since constellations on both polarizations are the same, it could be interpreted that $P_{\mathrm{L}}=2 \mathbb{E}\left\{|a|^{2}\right\}$, where $\mathbb{E}\left\{|a|^{2}\right\}=\mathbb{E}\left\{\left|a_{\mathrm{x}}\right|^{2}\right\}=\mathbb{E}\left\{\left|a_{\mathrm{y}}\right|^{2}\right\}$.

\section{Performarnce metrics}

In this section, two main parameters which are used for assessing system performance are discussed. Effective SNR after propagation of the light in the fiber-optic channel is defined as [22, Section IV]

$$
\mathrm{SNR}_{\mathrm{eff}}=\frac{P_{\mathrm{L}}}{\sigma_{\mathrm{eff}}^{2}}=\frac{P_{\mathrm{L}}}{\sigma_{\mathrm{ASE}}^{2}+\sigma_{\mathrm{NLI}}^{2}},
$$

where $\sigma_{\mathrm{ASE}}^{2}=N_{\mathrm{Span}} \cdot G \cdot \mathrm{NF} \cdot h \cdot v \cdot W$ is amlified spontaneous emission noise per two polarizations which induced by Erbium doped fiber amplifiers (EDFAs) used in the system. In this equation, $G$ and NF are gain and noise figure of each amplifier in each span, respectively, $h$ is Planck's constant, $v=\frac{C}{\lambda}$, where $C$ is the speed of the light, and $\lambda$ is the wavelength of the light in the COI. $W$ is the bandwidth of the transmitted signal. Since pulse shape of transmitted signal is assumed root-raised cosine (RRC) with a small roll-off factor $\beta$ which approximately is equal to sinc function in the time domain or rectangular in the frequency domain, $W$ is equal to $(1+\beta) R_{s}$, where $R_{s}$ is the baud rate of the transmission.

To dispart power dependency of NLI, one may rewrite Equation 6 as [22, Section IV-A]

$$
\mathrm{SNR}_{\mathrm{eff}}=\frac{P_{\mathrm{L}}}{\sigma_{\mathrm{ASE}}^{2}+P_{\mathrm{L}}^{3} \cdot \eta}
$$




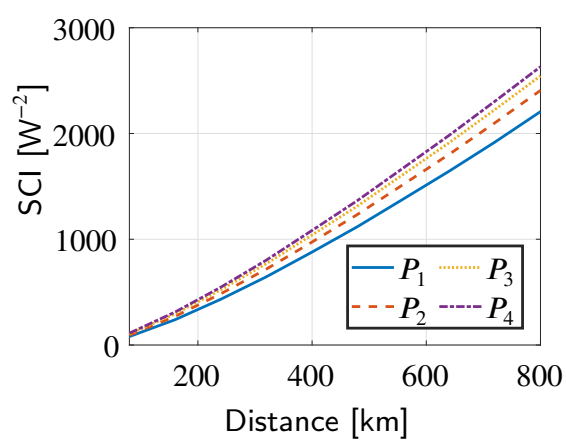

(a) SCI over distance

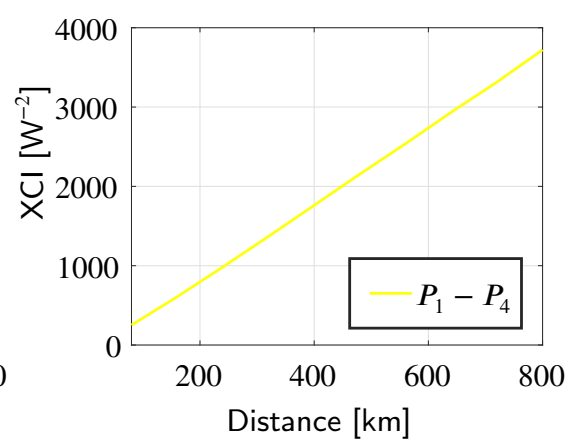

(b) XCI over distance

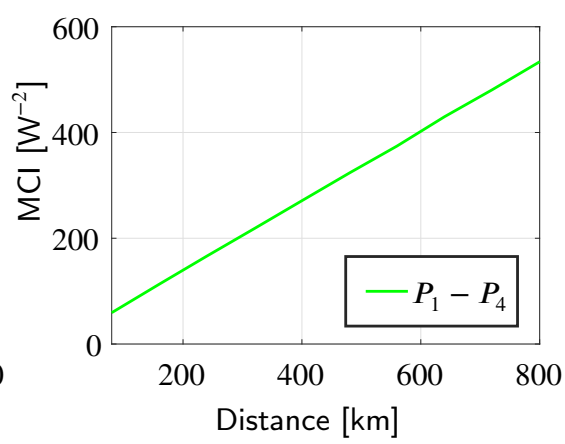

(c) MCI over distance

Figure 2: NLI terms for various PS schemes of Figure 1 applied to COI. These figures show the effect of PS on each term of $\mathrm{NLI}$. XCl and $\mathrm{MCl}$ are also affected by PS, but they are negligible that is approximately the same for all cases $P_{1}-P_{4}$.

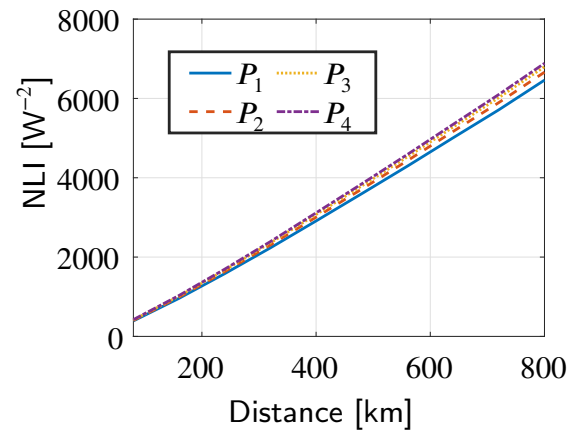

(a) NLI over distance

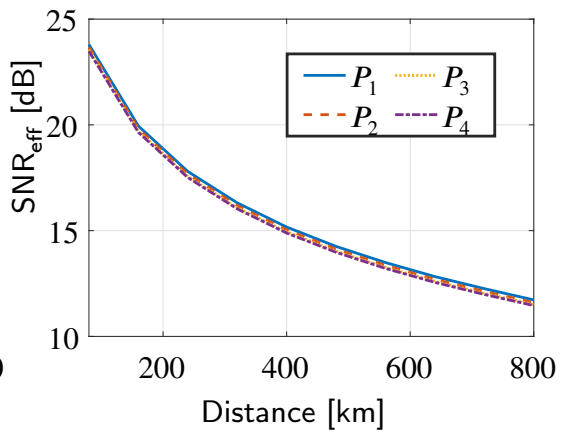

(b) effective SNR over distance

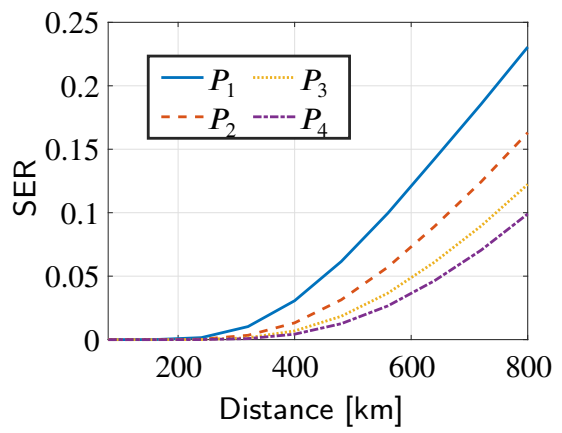

(c) SER over distance

Figure 3: NLI, Effective SNR, and SER for various PS schemes of Figure 1 applied to COI.

where $\eta$ is normalized NLI noise variance which does not depend on power. To find the optimum input power which maximizes the value of $\mathrm{SNR}_{\mathrm{eff}}, \frac{\partial \mathrm{SNR}_{\text {eff }}}{\partial P_{\mathrm{L}}}$ must be equal to zero which results in

$$
P_{\mathrm{L}}^{*}=\sqrt[3]{\frac{\sigma_{\mathrm{ASE}}^{2}}{2 \eta}},
$$

where $P_{\mathrm{L}}^{*}$ is the optimum input power that maximizes value of $\mathrm{SNR}_{\mathrm{eff}}$ and results in $\sigma_{\mathrm{NLI}}^{2}=\frac{\sigma_{\mathrm{ASE}}^{2}}{2}$ which is specially useful for validation of simulations.

We notice here that $\mathrm{SNR}_{\text {eff }}$ is not a proper tool for measuring transmission reach. In fact, it merely measures effective SNR which is a useful criterion in optical communications in general. But since it does not take into account discrete signal space concepts in SNR calculation, it can not account for the Euclidean distance change resulted by PS which is directly influencing transmission reach. To this end, one may use SER as a metric for measuring and comparing transmission reach of distinct PS schemes. SER of a communication system with uniform $M-$ QAM is calculated as [23, Equation 4.3-30]

$$
P_{e}^{M-\mathrm{QAM}} \approx 4 Q\left(\sqrt{\frac{3 \varepsilon_{s, \mathrm{avg}}}{(M-1) N_{0}}}\right),
$$

where $Q\left(\right.$.) denotes Q-function, i.e. $Q(x)=\frac{1}{\sqrt{2 \pi}} \int_{x}^{\infty} e^{-\frac{u^{2}}{2}} d u$.

In this paper, PM 16-QAM constellation is used where $\varepsilon_{s, \text { avg }}=\frac{5}{2} d_{\min }^{2}$ is the average energy of the constellation symbols in each polarization and $N_{0}$ is half of AWGN variance, i.e. $N_{0}=\frac{\sigma^{2}}{2}$. Here $\sigma^{2}$ is identical to $\sigma_{\text {eff }}^{2}$ which includes both ASE and NLI made by link parameters. So one may rewrite SER as

$$
P_{e}^{16-\mathrm{QAM}} \approx 4 Q\left(\sqrt{\frac{d_{\mathrm{min}}^{2}}{2 N_{0}}}\right)=4 Q\left(\frac{d_{\mathrm{min}}}{\sigma}\right),
$$

where $d_{\min }$ is the minimum distance or Euclidean distance between the points in the constellation diagram. Since symbols are transmitted simultaneously on both polarizations, we must compute SER for PM system in the form of

$$
\begin{aligned}
P_{e, \mathrm{PM}}^{M-\mathrm{QAM}} & =1-P_{C, \mathrm{PM}}^{M-\mathrm{QAM}}=1-\left(P_{C}^{M-\mathrm{QAM}}\right)^{2} \\
& =1-\left(1-P_{e}^{M-\mathrm{QAM}}\right)^{2}
\end{aligned}
$$

The same formula for the shaped scheme is obtained in section Appendix. 


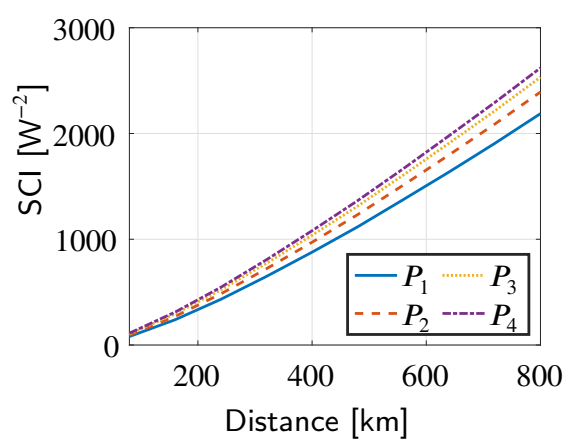

(a) SCI over distance

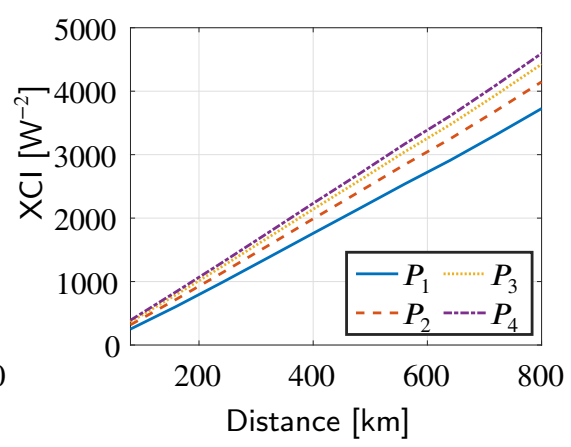

(b) XCI over distance

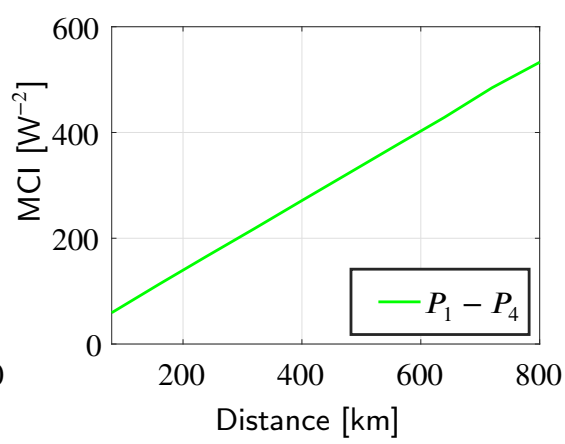

(c) MCI over distance

Figure 4: NLI terms for various PS schemes of Figure 1 applied to all WDM channels. These figures show the effect of PS on each term of $\mathrm{NLI}$. $\mathrm{MCl}$ is also affected by PS, but it is negligible that is approximately the same for all cases $P_{1}-P_{4}$.

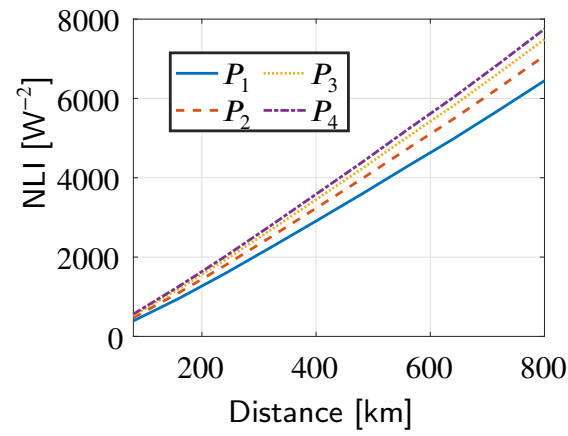

(a) NLI over distance

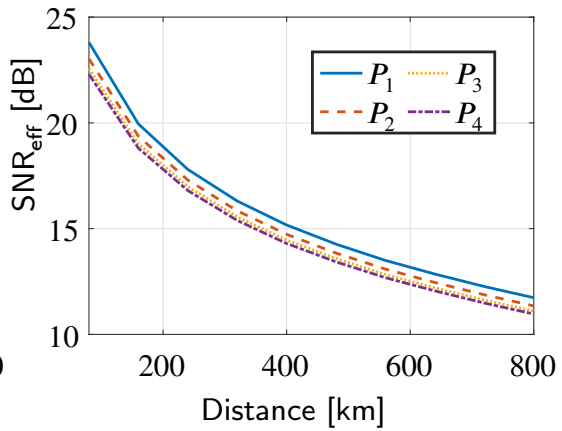

(b) effective SNR over distance

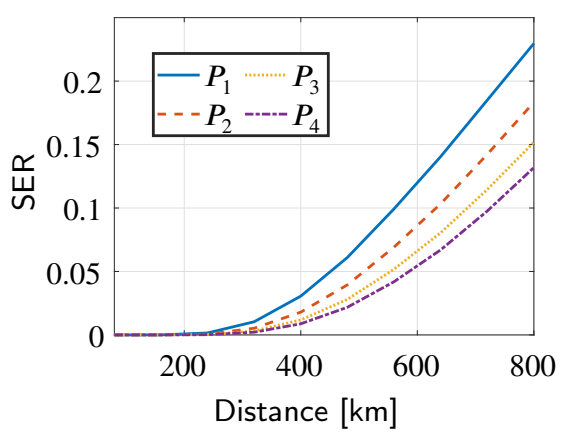

(c) SER over distance

Figure 5: NLI, effective SNR, and SER for various PS schemes of Figure 1 applied to all WDM channels.

\section{Numerical Results}

Comprehensive details of the simulation parameters and their values are available in Table 1 . The parameter values are chosen to be consistent with practical use. We then partition the results into two items where each of them corresponds to a different scenario of PS usage in WDM channels.

\subsection{Scenario I: PS in COI}

In this scenario, PS is solely applied to COI. It is shown that PS increases SCI and almost does not change XCI and MCI where can be seen in Figure 2. It is also revealed in Figure $3 \mathrm{a}$, Figure $3 \mathrm{~b}$ that for a fixed average power, PS increases NLI and decreases $S_{N R}$ eff a bit which is consistent with the theory and is expected according to Equation 7. It is also evident that PS reduces SER because increasing minimum distance of the constellation due to PS overcomes increasing NLI due to it which is depicted in Figure 3c. Indeed, PS causes an increase of up to $47.6 \%$ in the transmission reach.

\subsection{Scenario II: PS in all WDM channels}

In this scenario, PS is applied to all WDM channels. Same changes due to PS is happened, but it also increases $\mathrm{XCI}$ like as SCI and almost does not change MCI which is depicted in Figure 4. It also leads to more increase in NLI
Table 1

System and Simulation Parameters

\begin{tabular}{c|c} 
Parameter & Value \\
\hline Modulation & $16-$ QAM \\
Input PMF & uniform and nonuniform \\
Blocklength of CCDM & 21600 \\
Input launch power & $5 \mathrm{dBm}$ \\
Polarization & Dual \\
Symbol rate & $32 \mathrm{GBaud}$ \\
Pulse shape & RRC \\
RRC roll-Off & 0.01 \\
WDM channel number & 9 \\
WDM channel spacing & $1.05 \times$ Symbol Rate \\
Nonlinear coefficient $(\gamma)$ & $1.31 / \mathrm{W} / \mathrm{km}$ \\
Dispersion & $17 \mathrm{ps} / \mathrm{km} . \mathrm{nm}$ \\
Attenuation $(\alpha)$ & $0.22 \mathrm{~dB} / \mathrm{km}$ \\
Length per span & $80 \mathrm{~km}$ \\
Amplification & EDFA \\
EDFA noise figure & $5.5 \mathrm{~dB}$ \\
Laser wavelength $(\lambda)$ & $1550 \mathrm{~nm}$
\end{tabular}

and more decrease in effective SNR where can be seen in Figure 5a, Figure 5b, respectively, nevertheless PS still reduces SER because increasing minimum distance of the constellation due to PS is more prominent than increasing NLI 
due to it which is depicted in Figure 5c. In fact, an increase of up to $32.3 \%$ in the transmission reach is obtained.

It might be strange for the reader that NLI increases by PS. In a fixed average power, NLI increases by PS, because power is assumed to be fixed and the normalized value of NLI increases, so does NLI. It is worth mentioning that the NLI increase due to PS is almost not dependent to channel spacing value.

\section{Conclusions}

We have studied the effect of PS on NLI terms which are SCI, XCI, and MCI. Effective SNR and SER has been assessed in a multi-span WDM system in both cases of PS in the COI and PS in all WDM channels. It has been demonstrated that in the first case, NLI has $6.6 \%$ increase which is due to the effect of PS on SCI, while effective SNR has 0.27 $\mathrm{dB}$ decrease, and up to $47.6 \%$ reach increase is observed. In the second case, PS has more prominent role in increasing NLI due to its effect on SCI and XCI. It also leads to a 0.77 $\mathrm{dB}$ decrease in the effective SNR and up to $32.3 \%$ increase in the transmission reach. It has been manifested that the second case has more decrease in the effective SNR and it has less gain in the transmission reach due to PS which is not of interest. So PS in the COI is absolutely sufficient.

The benefit of the presented evaluation is confirming the fact that PS affects NLI terms differently, i.e., SCI and XCI terms which are dominant NLI terms, are more sensitive to PS, and MCI can be neglected for this kind of analysis. The other advantage is that in a point-to-point fiber-optic system, applying PS to the COI is enough. So one does not need to do it for all of the WDM channels. It simplifies the analysis and allows focusing on system parameters.

\section{Appendix}

To calculate SER of a shaped $M$-ASK or $M$ point pulse amplitude modulation (PAM) constellation, same as uniform scheme, constellation points are divided to two groups: inner and outer points. Probability of error for inner and outer points is computed as [23, Equations 4.3-2, 4.3-3], respectively. The symbol error probability is then given by

$$
\begin{aligned}
P_{e, M-\mathrm{PAM}} & =\sum_{m=1}^{M} P_{m} P[\text { error } \mid \text { m sent }] \\
& =\sum_{i=1}^{M-2} P_{i} P_{e i}+2 P_{o} P_{e o} \\
& =2 Q\left(\frac{d_{\min }}{\sqrt{2 N_{0}}}\right)\left[\sum_{i=1}^{M-2} P_{i}+P_{o}\right](M \geqslant 4),
\end{aligned}
$$

where $P_{i}$ and $P_{o}$ is the probability of each interior and exterior symbol, respectively. To find SER of $M-\mathrm{QAM}$ system, we have

$$
P_{c, M-\mathrm{QAM}}=P_{c, \sqrt{M}-\mathrm{PAM}}^{2}=\left(1-P_{e, \sqrt{M}-\mathrm{PAM}}\right)^{2}
$$

$$
P_{e, M-\mathrm{QAM}}=1-P_{c, M-\mathrm{QAM}}=1-\left(1-P_{e, \sqrt{M}-\mathrm{PAM}}\right)^{2},
$$

where

$$
\begin{gathered}
P_{e, \sqrt{M}-\mathrm{PAM}}=P_{e}=2 Q\left(\frac{d_{\min }}{\sqrt{2 N_{0}}}\right)\left[\sum_{i=1}^{\sqrt{M}-2} P_{i}+P_{o}\right] \\
(\sqrt{M} \geqslant 4)
\end{gathered}
$$

To compute SER for PM $M-\mathrm{QAM}$, we must compute 1 $\left(1-\left(1-\left(1-P_{e}\right)^{2}\right)\right)^{2}$ which results in $1-\left(1-P_{e}\right)^{4}$.

\section{Acknowledgement}

The authors would like to thank M. Ayaz for his fruitful comments on improving this paper.

\section{References}

[1] E. Agrell, M. Karlsson, A. R. Chraplyvy, D. J. Richardson, P. M. Krummrich, P. Winzer, K. Roberts, J. K. Fischer, S. J. Savory, B. J. Eggleton, M. Secondini, F. R. Kschischang, A. Lord, J. Prat, I. Tomkos, J. E. Bowers, S. Srinivasan, M. Brandt-Pearce, and N. Gisin, "Roadmap of optical communications," Journal of Optics, vol. 18, no. 6, p. 063002, may 2016. [Online]. Available: https://doi.org/10.1088/2040-8978/18/6/063002

[2] A. Alvarado, D. J. Ives, S. J. Savory, and P. Bayvel, "On the impact of optimal modulation and FEC overhead on future optical networks," Journal of Lightwave Technology, vol. 34, no. 9, pp. 2339-2352, 2016.

[3] J. Cho and P. J. Winzer, "Probabilistic constellation shaping for optical fiber communications," Journal of Lightwave Technology, vol. 37, no. 6, pp. 1590-1607, 2019.

[4] G. Böcherer, F. Steiner, and P. Schulte, "Bandwidth efficient and ratematched low-density parity-check coded modulation," IEEE Transactions on Communications, vol. 63, no. 12, pp. 4651-4665, 2015.

[5] H. Rabbani, G. Liga, V. Oliari, L. Beygi, E. Agrell, M. Karlsson, and A. Alvarado, "A general analytical model of nonlinear fiber propagation in the presence of kerr nonlinearity and stimulated raman scattering," arXiv: Signal Processing, 2019.

[6] P. Poggiolini, "The GN model of non-linear propagation in uncompensated coherent optical systems," Journal of Lightwave Technology, vol. 30, no. 24, pp. 3857-3879, Dec 2012. [Online]. Available: http://jlt.osa.org/abstract.cfm?URI=jlt-30-24-3857

[7] A. Carena, G. Bosco, V. Curri, Y. Jiang, P. Poggiolini, and F. Forghieri, "EGN model of non-linear fiber propagation," Optics Express, vol. 22, no. 13, pp. 16335-16362, Jun 2014. [Online]. Available: http://www.opticsexpress.org/abstract.cfm?URI= oe-22-13-16335

[8] F. R. Kschischang and S. Pasupathy, "Optimal nonuniform signaling for gaussian channels," IEEE Transactions on Information Theory, vol. 39, no. 3, pp. 913-929, 1993.

[9] R. Laroia, N. Farvardin, and S. A. Tretter, "On optimal shaping of multidimensional constellations," IEEE Transactions on Information Theory, vol. 40, no. 4, pp. 1044-1056, 1994.

[10] A. K. Khandani and P. Kabal, "Shaping multidimensional signal spaces. i. optimum shaping, shell mapping," IEEE Transactions on Information Theory, vol. 39, no. 6, pp. 1799-1808, 1993.

[11] S. A. Tretter, Constellation Shaping, Nonlinear Precoding, and Trellis Coding for Voiceband Telephone Channel Modems with Emphasis on ITU-T Recommendation V.34. USA: Kluwer Academic Publishers, 2002 . 
[12] L. Beygi, E. Agrell, J. M. Kahn, and M. Karlsson, "Coded modulation for fiber-optic networks: Toward better tradeoff between signal processing complexity and optical transparent reach," IEEE Signal Processing Magazine, vol. 31, no. 2, pp. 93-103, 2014.

[13] C. Pan and F. R. Kschischang, "Probabilistic 16-QAM shaping in WDM systems," Journal of Lightwave Technology, vol. 34, no. 18, pp. 4285-4292, 2016.

[14] G. Bosco, "Advanced modulation techniques for flexible optical transceivers: The rate/reach tradeoff," Journal of Lightwave Technology, vol. 37, no. 1, pp. 36-49, 2019.

[15] F. Buchali, F. Steiner, G. Böcherer, L. Schmalen, P. Schulte, and W. Idler, "Rate adaptation and reach increase by probabilistically shaped 64-QAM: An experimental demonstration," Journal of Lightwave Technology, vol. 34, no. 7, pp. 1599-1609, April 2016.

[16] T. Fehenberger, A. Alvarado, G. BÃúcherer, and N. Hanik, "On probabilistic shaping of quadrature amplitude modulation for the nonlinear fiber channel," Journal of Lightwave Technology, vol. 34, no. 21, pp. 5063-5073, 2016.

[17] T. Fehenberger, "Information rates of probabilistically shaped coded modulation for a multi-span fiber-optic communication system with 64QAM," Optics Communications, vol. 409, pp. 2 - 6, 2018, advances in modulation and DSP for optical transmission systems. [Online]. Available: http://www.sciencedirect.com/science/article/ pii/S0030401817306193

[18] P. Schulte and G. Böcherer, "Constant composition distribution matching," IEEE Transactions on Information Theory, vol. 62, no. 1, pp. 430-434, Jan 2016.

[19] G. Böcherer and B. C. Geiger, "Optimal quantization for distribution synthesis," IEEE Transactions on Information Theory, vol. 62, no. 11, pp. 6162-6172, 2016.

[20] P. Schulte, F. Steiner, and G. Böcherer, "shapecomm WebDM: Online constant composition distribution matcher," http://dm.shapecomm. de, Jul. 2017.

[21] R. E. Caflisch, "Monte Carlo and quasi-Monte Carlo methods," Acta numerica, vol. 7, pp. 1-49, 1998.

[22] P. Poggiolini, G. Bosco, A. Carena, V. Curri, Y. Jiang, and F. Forghieri, "The GN-model of fiber non-linear propagation and its applications," Journal of Lightwave Technology, vol. 32, no. 4, pp. 694-721, 2014.

[23] J. G. Proakis and M. Salehi, Digital Communications. Mc Graw Hill, 5th edition, 2008. 